\title{
Functional alterations of myeloid cells during the course of Alzheimer's disease
}

\author{
Aaron D. Thome, Alireza Faridar, David R. Beers, Jason R. Thonhoff, Weihua Zhao, Shixiang Wen, Belen Pascual, \\ Joseph C. Masdeu and Stanley H. Appel*
}

\begin{abstract}
Background: Neuroinflammation is a hallmark of neurodegenerative disease and a significant component of the pathology of Alzheimer's disease (AD). Patients present with extensive microgliosis along with elevated proinflammatory signaling in the central nervous system and periphery. However, the role of peripheral myeloid cells in mediating and influencing AD pathogenesis remains unresolved.

Methods: Peripheral myeloid cells were isolated from peripheral blood of patients with prodromal $\operatorname{AD}(n=44)$, mild AD dementia $(n=25)$, moderate/severe AD dementia $(n=28)$, and age-matched controls $(n=54)$. Patients were evaluated in the clinic for AD severity and categorized using Clinical Dementia Rating (CDR) scale resulting in separation of patients into prodromal AD (CDR0.5) and advancing forms of AD dementia (mild-CDR1 and moderate/severe-CDR2/3). Separation of peripheral myeloid cells into mature monocytes or immature MDSCs permitted the delineation of population changes from flow cytometric analysis, RNA phenotype analysis, and functional studies using T cell suppression assays and monocyte suppression assays.
\end{abstract}

Results: During stages of AD dementia (CDR1 and 2/3) peripheral myeloid cells increase their pro-inflammatory gene expression while at early stages of disease (prodromal AD—CDR0.5) pro-inflammatory gene expression is decreased. MDSCs are increased in prodromal AD compared with controls (16.81\% vs $9.53 \%)$ and have markedly increased suppressive functions: $42.4 \%$ suppression of activated monocyte-produced IL- 6 and $78.16 \%$ suppression of T cell proliferation. In AD dementia, MDSC populations are reduced with decreased suppression of monocyte IL-6 (5.22\%) and T cell proliferation (37.61\%); the reduced suppression coincides with increased pro-inflammatory signaling in AD dementia monocytes.

Conclusions: Peripheral monocyte gene expression is pro-inflammatory throughout the course of AD, except at the earliest, prodromal stages when pro-inflammatory gene expression is suppressed. This monocyte biphasic response is associated with increased numbers and suppressive functions of MDSCs during the early stages and decreased numbers and suppressive functions in later stages of disease. Prolonging the early protective suppression and reversing the later loss of suppressive activity may offer a novel therapeutic strategy.

Keywords: Alzheimer's disease, Inflammation, Myeloid cells, Monocyte, Microglia, Myeloid-derived suppressor cells

\footnotetext{
* Correspondence: sappel@houstonmethodist.org

Department of Neurology, Houston Methodist Neurological Institute, 6560

Fannin St. Suite 802, Houston, TX 77030, USA
}

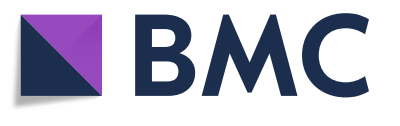

(c) The Author(s). 2018 Open Access This article is distributed under the terms of the Creative Commons Attribution 4.0 International License (http://creativecommons.org/licenses/by/4.0/), which permits unrestricted use, distribution, and reproduction in any medium, provided you give appropriate credit to the original author(s) and the source, provide a link to the Creative Commons license, and indicate if changes were made. The Creative Commons Public Domain Dedication waiver (http://creativecommons.org/publicdomain/zero/1.0/) applies to the data made available in this article, unless otherwise stated. 


\section{Background}

Alzheimer's disease (AD) is the most common neurodegenerative disease and is characterized by cognitive impairment, amyloid- $\beta(\mathrm{A} \beta)$ deposition, and neurofibrillary tangle formation [1-5]. Increasing evidence suggests that immune mechanisms contribute to the pathogenesis of AD including reactive microgliosis in postmortem samples, increased microglial activation marker, translocator protein (TSPO), binding on positron emission tomography (PET), [6] and increased pro-inflammatory cytokines such as interleukin (IL)-6, IL-1 $\beta$, TNF, and IFN- $\gamma$ in the cerebrospinal fluid and serum [7-11]. Thus, neuroinflammation is potentially a target for immunomodulatory therapies [12, 13].

Genome-wide association studies implicate immune system dysfunction, particularly in myeloid-derived cells, as immune-related genes coding for triggering receptor expressed on myeloid cells 2 (TREM2) and CD33 confer increased risk for AD susceptibility [14-18]. Current studies focus on microglia, resident myeloid cells of the brain, throughout AD pathogenesis, but recent literature in neurodegenerative diseases suggest extensive neuro-immune cross-talk between the brain and peripheral immune system [19-22]. This cross-talk may derive either directly or indirectly from peripheral immune cells in the presence of a compromised blood brain barrier (BBB) such as in neurodegenerative disease [23-25]. Additionally during inflammatory insult and microglial depletion, peripheral macrophage engraftment into the CNS was observed with these cells retaining a distinct and lasting transcriptional and functional identity [26]. Thus peripheral immune myeloid cells could modulate disease progression and outcomes in the CNS. The accessibility of these peripheral myeloid cells, and the lack of accessibility to CNS microglia, prompted a detailed examination of blood monocyte populations during the pathoprogression of $\mathrm{AD}$.

Immature and mature monocytes, here-after denoted as "peripheral myeloid cells," originate from hematopoietic stem cells and mature into peripheral monocytes with the capability of differentiating into macrophages once they enter tissue parenchyma [27-31]. Changes and shifts in peripheral myeloid populations are indicators of disease onset and progression for a multitude of diseases; the pro-inflammatory phenotypes have direct effects on their specific disease [32-40]. A detailed analysis of peripheral monocyte population and phenotype changes have not been documented thoroughly in AD progression.

Myeloid-derived suppressor cells (MDSCs) are immature myeloid cells that exhibit robust suppressive function on $\mathrm{T}$ cell proliferation and mature myeloid cell function which has made them a target of multiple immunomodulatory therapies [41-44]. Chronic AD immune signaling provides a practical mechanism where MDSC activation and recruitment potentially promotes an immunosuppressive environment [45]. However, the actual role of MDSCs in AD pathology and progression remains unexplored. A detailed investigation into the role and function of mature and immature peripheral myeloid cells could provide insight into mechanisms of their direct involvement in AD pathogenesis as well as provide clues as to the changes in CNS myeloid cells through disease progression.

In this study, we investigated peripheral myeloid populations and their pro-inflammatory gene expression throughout the course of AD compared with age-matched controls. We also investigated changes in a novel and previously unrecognized cell type in AD, MDSCs, to determine their phenotypes during course of disease. Understanding the role of peripheral myeloid cells in AD-related inflammation and mechanisms of their immune suppression may lead to novel therapeutic interventions for the successful treatment of $\mathrm{AD}$.

\section{Methods}

Patient recruitment and AD-defined population

$\mathrm{AD}$ patients and aged-matched healthy controls were recruited into the study from the Houston Methodist Nantz National Alzheimer's Center (NNAC) based on National Institute on Aging-Alzheimer's Association (NIA-AA) diagnostic criteria [46, 47]. The study was approved by the Houston Methodist IRB and all participants signed informed consent. Clinical evaluations for prodromal AD (CDR0.5) $(n=44)$, mild AD dementia (CDR1) $(n=25)$, moderate/severe AD dementia (CDR2/ 3) $(n=28)$, and age-matched, healthy controls $(n=54)$ were made under the direction of Dr. Joseph Masdeu and colleagues at the NNAC. Staging of dementia severity was based on the Clinical Dementia Rating (CDR) Scale (Table 1).

\section{Immune cell isolation}

Immune cells were isolated from peripheral blood of participants using Lymphoprep density gradient (STEMCELL) followed by Human Pan Monocyte Isolation Kit (Miltenyi Biotec) for negative selection of human monocytes. Further isolation using anti-HLA-DR microbeads (Miltenyi Biotec) allowed for the separation of HLA-DR+, mature monocytes, and HLA-DR-, immature MDSCs (Additional file 1: Table S1). T responder cells were isolated from the PBMC pool using CD4+ CD25+

Table 1 Patient demographics

\begin{tabular}{lllll}
\hline Diagnosis & CDR & Number & M,F & Age, yr. \pm SD \\
\hline Control & CDR 0 & 54 & 30,24 & $69.81 \pm 6.52$ \\
Prodromal AD & CDR0.5 & 44 & 21,23 & $73.57 \pm 6.57$ \\
Mild AD Dementia & CDR1 & 25 & 10,15 & $74.48 \pm 7.98$ \\
Moderate/Severe AD Dementia & CDR2/3 & 28 & 9,19 & $71.29 \pm 8.52$ \\
\hline
\end{tabular}


regulatory $\mathrm{T}$ Cell Isolation Kit (Miltenyi Biotec) to obtain CD4 + CD25- cells.

\section{Flow cytometry}

Fluorescent immune cell probes for the delineation of monocyte subsets included anti-human CD14-V450, anti-human CD16-FITC, anti-human HLA-DR-PerCPCy5.5, anti-human CD33-APC, anti-human CD11b-PE, and IgG isotype controls (ebioscience or BD Biosciences). Cells were counted using an LSRII flow cytometer with the data was analyzed with BD FACSDIVA software. The submitted flow cytometry gating paradigm (Additional file 2: Figure S2) depicts cell populations for analysis of mature monocyte populations and the immature MDSC population.

\section{RNA purification, RT-PCR analysis, and Nanostring}

RNA was extracted from immune cell populations and in vitro cell experiments using Trizol reagent followed by Direct-zol RNA MiniPrep Kit (Zymo Research). Quantitative RT-PCR (qRT-PCR) experiments were performed using a One-Step RT-PCR kit with SYBR Green and run using Bio-Rad iQ5 Multicolor Real-Time PCR Detection Systems. Primers for the study were purchased from BioRad and the relative expression level of each mRNA was calculated using the $\Delta \Delta \mathrm{Ct}$ method with normalization to $\beta$-actin and relative to control samples. RNA sample QC and Nanostring experiments were performed using Baylor College of Medicine Genomic and RNA Profiling Core. Monocyte RNA was run on the nCounter Human Inflammation Panel (Nanostring, Human v2). The panel consisted of 255 human inflammation-related genes with 15 internal reference genes. Analysis of data QC, normalization, and differential gene expression was performed using nSolver analysis software provided by the company. Normalized data counts generated from Nanostring experiment provided in Additional file 3: Figure S9.

\section{T cell suppression assays}

Responder T cells and MDSCs were isolated using Miltenyi Biotec microbeads as previously described. CD4+ T responder cells were placed in a 96 well plate at a density of $50 \mathrm{k}$ cells per well followed by co-culture of MDSCs at a ratio of 1:1, 1:1/2, and 1:1/4 (T cell: MDSC). CD3/CD28 $\mathrm{T}$ cell stimulation reagent is then added to the co-culture for five days followed by addition of tritium (Miltenyi). Proliferation measured via tritium incorporation.

\section{Myeloid cell suppression assays}

Our lab has recapitulated protocols for the generation of mature monocyte cells from induced pluripotent stem cells (iPS cells). Yanagimachi et al. describes a detailed protocol and discussion of the process [48]. Isolation and polarization of CD14 cells into pro-inflammatory "M1" cells can be found in the culturing paradigm (Additional file 4: Figure S4). Briefly, iPSC-derived macrophages are grown from a control line and polarized to be pro-inflammatory. We then use MDSCs isolated from patients to co-culture with the pro-inflammatory iPSC-derived macrophages overnight $(18 \mathrm{~h})$ to look at suppressive function of the MDSCs on myeloid cells. We collected RNA from co-cultured pro-inflammatory macrophages for transcript analysis and cultured media for ELISA protein changes (Invitrogen).

\section{Results}

Peripheral myeloid cells have increased pro-inflammatory gene expression in AD dementia but significantly decreased expression in prodromal AD

RNA isolated from the blood-derived peripheral myeloid cells were analyzed using Nanostring chip and qRT-PCR to determine their inflammatory profiles. We detected increased pro-inflammatory RNA in AD dementia patients (CDR1, 2, 3) with increased expression of IL-6, IL-1 $\beta$, NLRP3, TNF, IL-18, and HLA-DRA, among others. (Fig. 1a). There was little to no increase in inflammation-associated RNA signatures between controls and prodromal AD (CDR0.5) patient cells (Fig. 1b). In fact, there were decreased RNA levels of C-reactive protein (CRP), CXCR4, CCL11, and complement system components $(\mathrm{C} 1 \mathrm{~s}$ and $\mathrm{C} 8 \mathrm{a})$ in prodromal $\mathrm{AD}$ cells compared with controls. Follow-up analysis from additional patients using qRT-PCR corroborated the Nanostring data showing decreased expression of IL- 6 and IL- $1 \beta$ in CDR0.5 peripheral myeloid cells but increased expression of these two cytokines in CDR1 and CDR2/3 peripheral myeloid cells (Fig. 1c). Additionally, anti-inflammatory IL-10 expression, as determined by qRT-PCR, was increased in CDR0.5 peripheral myeloid cells compared with controls but not in samples from CDR1 or CDR2/3 patients (Fig. 1c).

Surface level expression of HLA-DR, up-regulated during immune cell activation to promote pro-inflammatory signaling, was increased on mature monocytes from CDR1 and CDR2/3 compared with both controls and CDR0.5 (Fig. 1d). CD33 expression which is known to inhibit activation and cytokine release from myeloid cells was increased on cells from CDR0.5 patient monocytes but not on cells from controls and CDR1 and CDR2/3 patients (Fig. 1e). Gender and age of the controls and patients were not confounding variables in these experiments (Additional file 5: Figure S3 and Additional file 6: Figure S6).

\section{Alterations in mature and immature monocyte populations through $A D$ progression}

We observed a progressive decline of the classical monocytes $\left(\mathrm{CD} 14^{+} \mathrm{CD} 16^{-}\right)$with increasing $\mathrm{AD}$ burden 

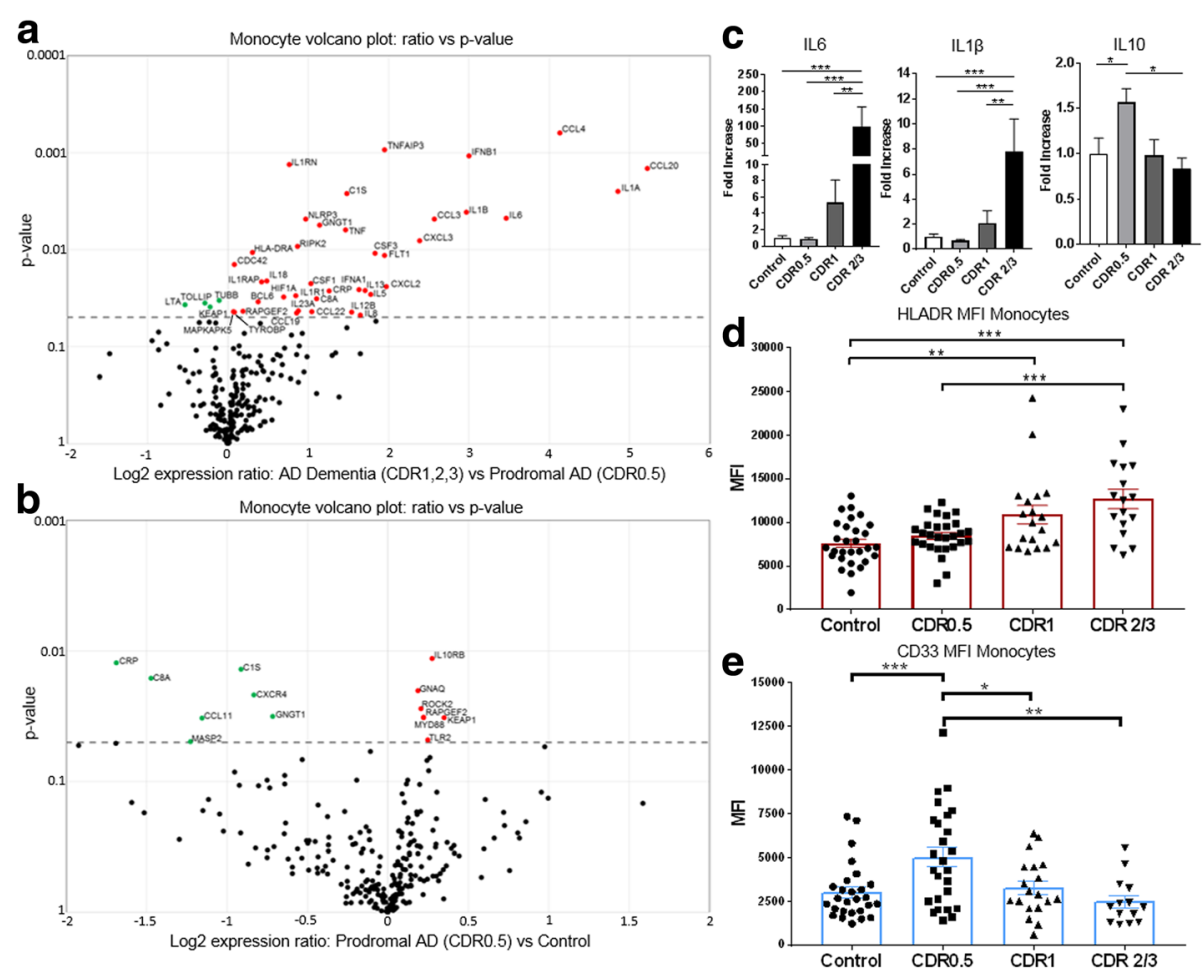

Fig. 1 Peripheral myeloid cells are pro-inflammatory in AD dementia but suppressed/anti-inflammatory in prodromal AD. Volcano plots showing log2 expression ratios vs $p$-values of $n C$ Counter human inflammation v2 panel for $(\mathbf{a})$ AD dementia $(C D R 1,2,3)$ vs prodromal $A D(C D R 0.5)$ and $(\mathbf{b})$ Prodromal AD (CDR0.5) vs Control (AD Dementia, $n=10$; Prodromal $A D, n=6 ; C n=8$ ). Analysis and statistics run using $n$ Solver 3.0 analysis software. (c) Upregulation of pro-inflammatory gene expression of IL-6 and IL-1 $\beta$ in peripheral myeloid cells in CDR1 and $2 / 3$ while IL-10 is increased in CDR0.5 peripheral myeloid cells ( $C n=20$, CDR0.5 $n=20$, CDR1 $n=8, C D R 2 / 3 n=10)$. Numbers shown as averages \pm SEM and with one-way ANOVA with Tukey's post hoc test. d Flow cytometric median fluorescence intensity of pro-inflammatory HLADR is increased on the surface of CDR1 and CDR2/3 peripheral myeloid cells while (e) inhibitory motif, CD33, is increased on the surface of CDR0.5 peripheral myeloid cells and decreases with increasing AD burden. Numbers shown as averages \pm SEM and with one-way ANOVA with Tukey's post hoc test (C $n=30, \operatorname{CDR} 0.5 n=27, \mathrm{CDR} 1$ $n=17, \mathrm{CDR} 2 / 3 n=13)$. $P$-values are ${ }^{*} p<0.05,{ }^{* *} p<0.01$, and ${ }^{* * *} p<0.001$

(Fig. 2a) leading to a redistribution of the populations to intermediate $\left(\mathrm{CD} 14^{+} \mathrm{CD} 16^{+}\right)$and non-classical populations $\left(\mathrm{CD} 14^{\text {low }} \mathrm{CD} 16^{+}\right)$. Intermediate populations in CDR0.5, 1 , and $2 / 3$ were increased compared with controls (Fig. 2b). CDR1 and CDR2/3 non-classical populations were increased compared with controls (Fig. 2c). Using $\mathrm{CD}_{14}{ }^{+}, \mathrm{HLA}^{-} \mathrm{DR}^{-}, \mathrm{CD}_{11 \mathrm{~b}^{+}}$, and $\mathrm{CD}^{+} 3^{+}$as cell surface marker for MDSCs, there was an increased number of MDSCs in CDR0.5 (16.81\%) and CDR1 (17.74\%) patients compared with age-matched controls $(9.53 \%)$. CDR2/3 MDSC levels (9.64\%) were similar to control levels (Fig. 2d). Gender and age did not confound monocyte population changes (Additional file 7: Figure S5).

\section{Pro-inflammatory responses of monocytes from $A D$ dementia patients and anti-inflammatory responses of MDSCs from prodromal AD patients}

Following separation of MDSCs from monocytes, RNA analysis demonstrated increases in pro-inflammatory signaling in monocytes from AD dementia patients. Specifically, there were increased mRNA expression of IL-6,
IL-1 $\beta$, and TNF in mature monocytes isolated from CDR1 and CDR2/3 patients when compared with controls (Fig. 3a). Additionally, there was a trend for a decrease in IL-6 transcript from CDR0.5 patient monocytes while the IL-1 $\beta$ and TNF were not different than controls. In evaluating anti-inflammatory signaling in MDSC populations, IL-10 and IL-13 transcripts were increased in CDR0.5 but then decreased as the disease progressed to CDR1 and CDR2/3 (Fig. 3b). Interestingly, anti-inflammatory TGF- $\beta$ was increased in mature monocytes from CDR0.5 patients but not from the monocytes of later stages.

\section{Pro-inflammatory myeloid cells and T cell proliferation are suppressed by prodromal AD MDSCs but not AD dementia MDSCs}

We investigated the suppressive capacity of MDSCs on a pro-inflammatory macrophage population. We co-cultured patient-isolated MDSCs with pro-inflammatory iPSC-derived macrophages overnight to examine attenuation of pro-inflammatory IL-6 transcript and protein. MDSCs from CDR0.5 patients attenuated IL-6 transcript expression 

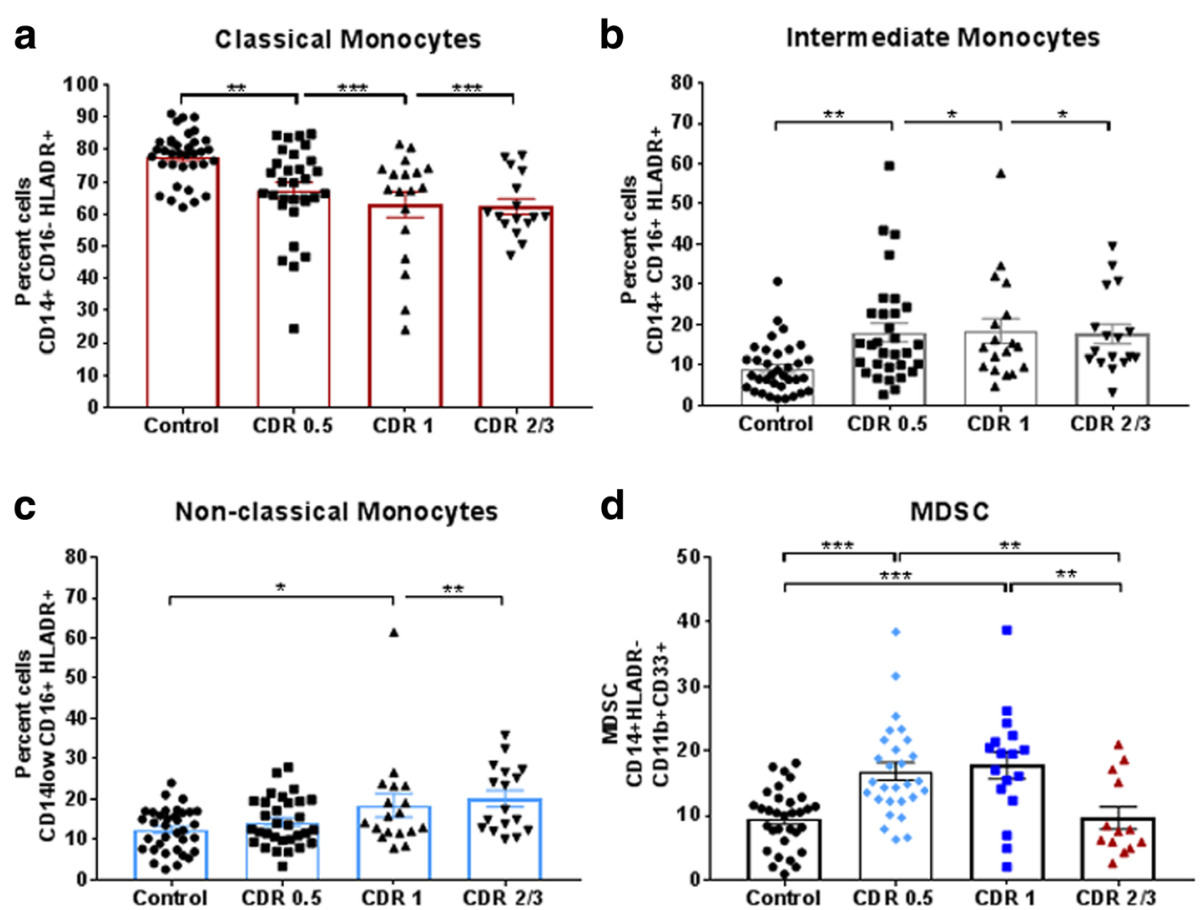

Fig. 2 Monocyte populations shift as AD burden increases. Monocyte population changes via flow cytometric analysis of CD14 and CD16 expression on HLADR+ myeloid immune cells. Decreases in (a) classical monocytes (CD14+ CD16-) with increasing disease burden with a redistribution of monocytes into (b) intermediate (CD14+CD16+) and (c) non-classical populations (CD14low CD16+). Numbers shown as averages \pm SEM and with one-way ANOVA with Tukey's post hoc analysis (Control $n=35, \operatorname{CDR} 0.5 n=31, \operatorname{CDR} 1 n=18, \operatorname{CDR} 2 / 3 n=17$ ). Increased MDSC populations (d) in CDR0.5 and CDR1 patients compared with controls as percent of total CD14 cell population. MDSC numbers fall to control levels in CDR2/3 patients (Control $n=32$, CDR0.5 $n=28, \operatorname{CDR} 1 n=17, \operatorname{CDR} 2 / 3 n=13$ ). Numbers shown as averages \pm SEM and with oneway ANOVA with Tukey's post hoc test. $P$-values are ${ }^{*} p<0.05$, ${ }^{* *} p<0.01$, and ${ }^{* *} p<0.001$

(42.4\%) compared with control MDSCs (22.09\%) (Fig. 4a). This attenuation of IL- 6 expression decreased in CDR1 (17.33\%) and CDR2/3 (5.22\%) patients. In examination of the co-cultured media, we find decreased IL-6 protein when we co-culture pro-inflammatory macrophages (alone-18,208 pg/mL IL-6) with CDR0.5 MDSCs (6154 pg/ $\mathrm{mL})$. This suppressive effect decreases as patients advance to CDR1 (10,856 $\mathrm{pg} / \mathrm{mL})$ and later into CDR 2/3 $(16,348 \mathrm{pg} / \mathrm{mL})$. Control MDSCs in our experiment were able to reduce pro-inflammatory IL-6 output to $12,791 \mathrm{pg} /$ $\mathrm{mL}$ while MDSCs alone did not produce IL-6 protein. Gender and age did not affect suppressive capacity of control or patient MDSCs (Additional file 8: Figure S7). Our RNA expression data from isolated mature monocytes and MDSCs displayed increased IL-10 transcript from CDR0.5 MDSCs. Adding an IL-10 neutralizing antibody (IL10ab) to the MDSC: M1 cultures at $5 \mu \mathrm{g} / \mathrm{mL}$ significantly reduced suppression of IL-6 transcripts (Fig. 4c), suggesting that secretion of IL-10 contributes to the MDSC-mediated suppression.

The suppression profiles of MDSCs were next examined when co-cultured with $\mathrm{CD}^{+}{ }^{+}$Tresp. CDR0.5 and CDR1 MDSCs showed increased suppression of autologous Tresp proliferation compared with the suppression of control MDSCs. Similar to the decreased numbers of MDSCs, there was decreased MDSC suppressive function in CDR2/3 compared with all groups. At a 1:1 ratio (Tresp: MDSC), CDR0.5 and CDR1 yielded $79.03 \%$ and $61.81 \%$ suppression, respectively, compared with control $55.7 \%$ suppression while CDR2/3 MDSCs dropped to 37.61\% (Fig. 4d, e). When examining the expression of arginase-1 (Arg1) as a mechanism to block T cell proliferation, Arg1 expression was increased in CDR0.5 MDSCs compared with controls and subsequently decreased in CDR1 and then in CDR2/3 patients (Fig. 4f). $\mathrm{T}$ cell suppression by MDSCs positively correlated with myeloid cell suppression; patients with early disease possess increased suppressive function while suppressive function is progressively impaired as disease advances (Additional file 9: Figure S8).

\section{Discussion}

The analyses of peripheral myeloid cells in $\mathrm{AD}$ patients provided three important findings. First, pro-inflammatory gene-expressing phenotypes of peripheral monocytes are increased in CDR1,2,3 patients. Second, pro-inflammatory gene-expressing phenotypes of peripheral monocytes are decreased in CDR0.5 patients. Third, MDSCs are increased 


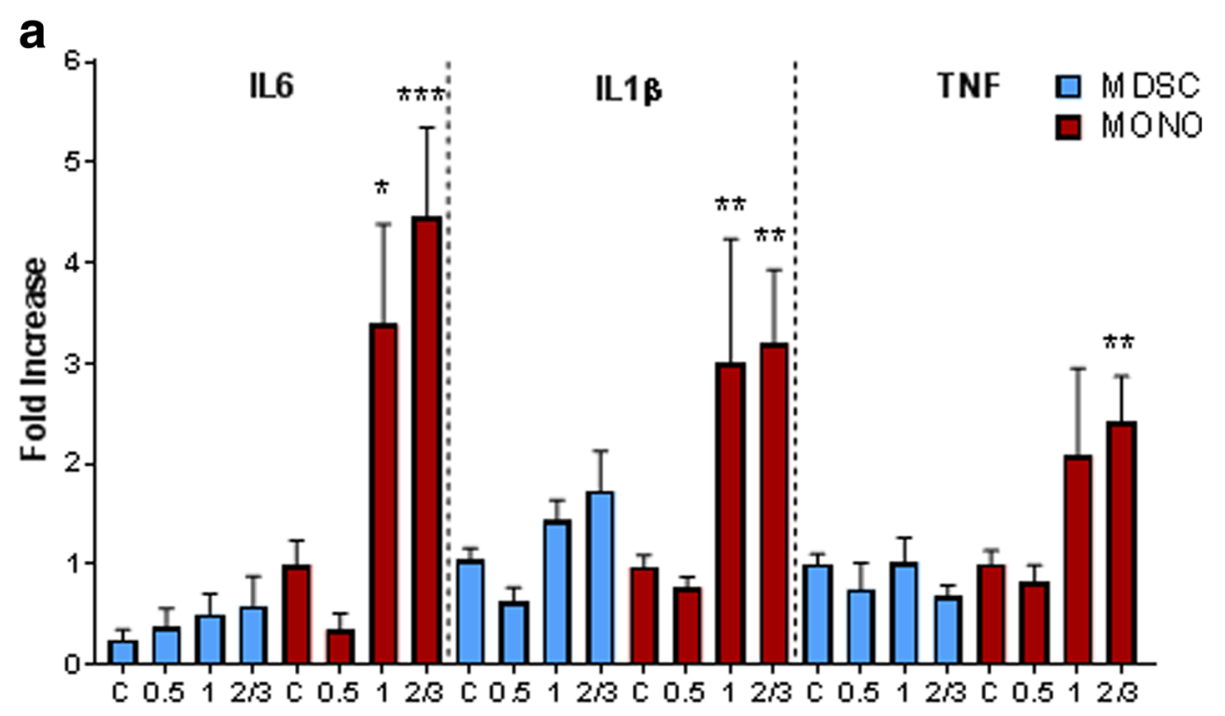

b

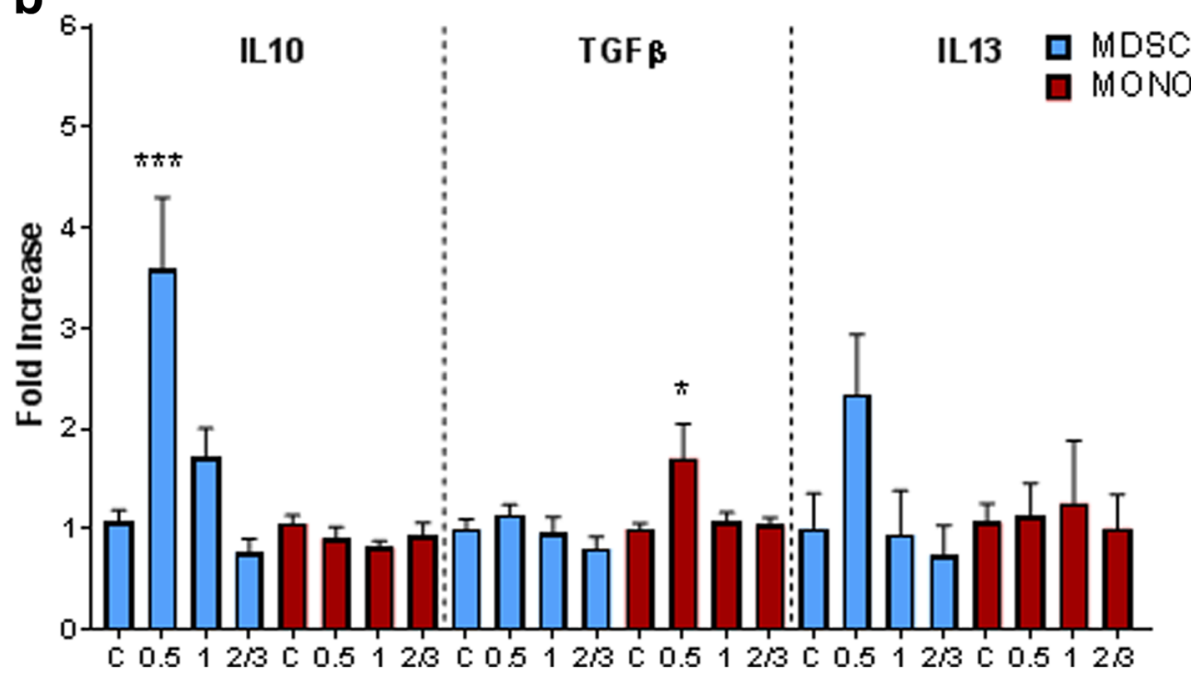

Fig. 3 Mature monocytes are pro-inflammatory in AD dementia while MDSCs are anti-inflammatory in prodromal AD. Isolated mature monocytes and MDSCs displayed differential inflammatory gene expression profiles. a Mature monocytes from CDR1 and CDR2/3 display increased pro-

inflammatory cytokine expression via IL-6, IL-1 $\beta$, and TNF. b MDSCs from CDR0.5 patients have increased anti-inflammatory IL-10 and IL-13 expression. TGF $\beta$ transcripts were increased in CDR0.5 monocytes. One-way ANOVA with Tukey's post hoc analysis (Control $n=13-14, \operatorname{CDR} 0.5 n=8, C D R 1 n=7$, CDR2/3 $n=10)$. P-values are ${ }^{*} p<0.05,{ }^{* *} p<0.01$, and ${ }^{* * *} p<0.001$

in number and suppressive function in CDR0.5 and decreased in CDR2/3. MDSC from CDR0.5 suppressed Tresp proliferation and iPSC-derived pro-inflammatory myeloid cells in vitro more effectively than control MDSCs. MDSCs from CDR $2 / 3$ patients had significantly decreased suppressive function for both Tresp proliferation and pro-inflammatory myeloid cells.

Activation of myeloid cells has been extensively studied in humans and animal models of neurodegeneration, including $\mathrm{AD}$, with much of the focus on brain microglia which are difficult to study directly during the course of disease. Peripheral myeloid cells have similar functions to brain microglia including chemotaxis, phagocytosis, and cytokine expression but constitute a cell population that can be collected and studied through simple, minimally-invasive methods [28, 49-51]. Activation of immune cells can be assessed using flow cytometry by analyzing HLA-DR, a MHC class II cell surface receptor that is upregulated upon antigen presenting cell activation. HLA-DR expression was increased on mature monocytes from CDR1 and CDR2/3 patients when compared with controls and CDR0.5 which matches the pro-inflammatory gene expression seen in these patients. CDR0.5 monocytes did not exhibit pro-inflammatory gene expression but displayed increased inhibitory CD33 cell surface expression compared with controls and this expression diminished in CDR1 patients while decreasing below control values in CDR2/3 patients. 

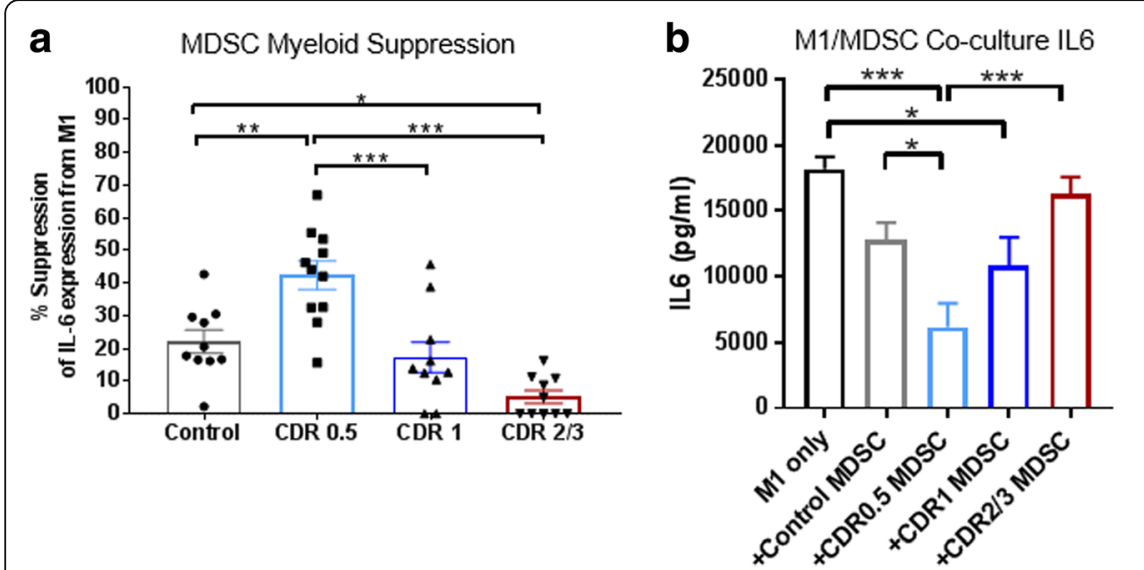

C Suppression changes IL10ab
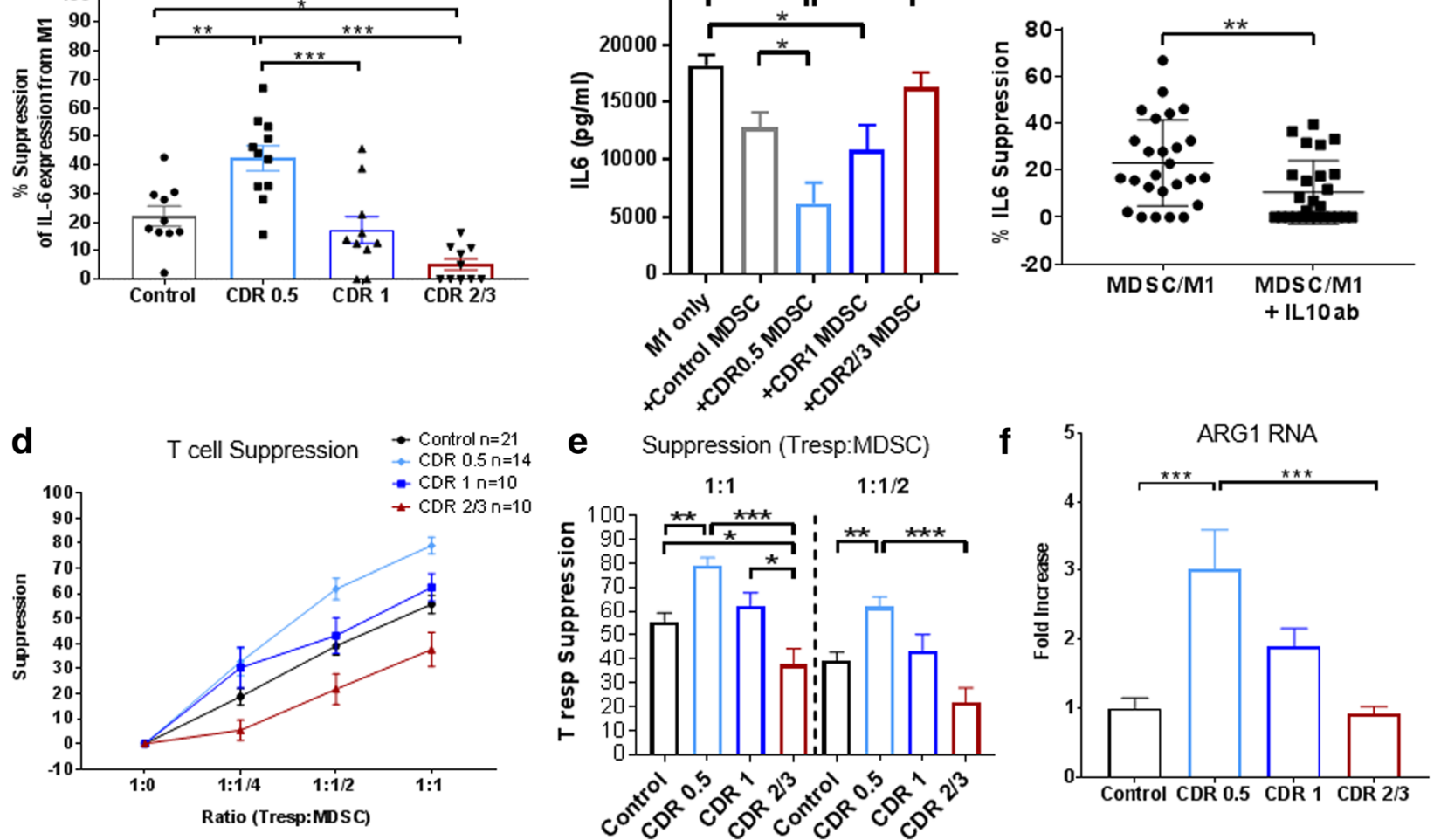

Fig. 4 Stage-specific MDSC suppression of pro-inflammatory myeloid cells and T cell proliferation. Isolated patient MDSCs are co-cultured overnight (18 h) at 1:1 ratio with iPSC-generated M1 pro-inflammatory macrophages. a Production of IL-6 transcripts were highly suppressed by MDSCs from CDR0.5 patients while that suppression decreases as the patient advances to CDR1 and CDR2/3. (Control $n=10$, CDR0.5 $n=11$, CDR1 $n=10$, and CDR2/3 $n=10)$. b Conditioned media from PPSC-M1 and patient MDSC co-cultures were analyzed via ELISA for changes in IL-6 protein. Changes in protein levels mirrored the IL-6 RNA suppression with CDR0.5 MDSCs causing a decrease in IL-6 protein production by M1 cells. These suppressive effects tapered off with advancing disease condition (Control $n=6, \operatorname{CDR0.5} n=6, \operatorname{CDR} 1 n=4$, and CDR2/3 $n=5$ ). c Addition of $5 \mu \mathrm{g} / \mathrm{mL}$ neutralizing antibody for IL-10 reduces the IL-6 suppression in our MDSC: M1 assays. Numbers shown as averages \pm SEM and statistics utilized were one-way ANOVA with Tukey post hoc $(\mathbf{a}, \mathbf{b})$ and student's t-test (c). Isolated MDSCs were co-cultured with T responder cells at ratios of 1:1/4, 1:1/2, and 1:1 and stimulated for proliferation. d, e CDR0.5 MDSCs show hypersuppression of T cell proliferation compared with other groups. Late CDR2/3 MDSCs lost their T cell proliferation suppression below that of controls (Control $n=21, \operatorname{CDR} 0.5 n=14, \operatorname{CDR} 1 n=10$, and CDR2/3 $n=10)$. f Arg1 expression in isolated MDSCs increase in CDR0.5 but decrease with increasing AD burden (Control $n=12, \mathrm{CDR} 0.5 n=$ $8, \operatorname{CDR} 1 n=8, C D R 2 / 3 n=10)$. Numbers shown as averages \pm SEM with one-way ANOVA with Tukey's post hoc analysis. $P$-values are ${ }^{*} p<0.05$, ${ }^{* *} p<0.01$, and ${ }^{* * *} p<0.001$

While some studies implicate differential activation and polarization of immune cells due to the age and gender of the patient, we did not observe any significant effects contributed by these factors in our control or AD datasets. Advanced studies into gender and age-specific contributions to neurodegenerative disease are warranted.

The peripheral pattern of immune cell deactivation and reactivation is in accord with the biphasic immune marker profile reported in a study of CSF immune makers in AD [52]; decreases in immune signaling were noted early in the course of disease and a strong increase in immune signaling was noted in later stages of disease. Additionally, a longitudinal imaging study of $\mathrm{AD}$ patients found initial microglial activation upon diagnosis followed by a reduction of activation as patients went through mild cognitive impairment stages. As patients progressed into later stages of $\mathrm{AD}$, microglial activation increased again suggesting that there might be two activation stages; the first being a protective activation state while the later peak being a more pro-inflammatory and destructive process [53].

Many studies utilize changes and shifts in monocyte populations to identify and predict inflammatory disease and progression in a multitude of inflammation-based disorders [38-40]. Previous AD studies suggest monocyte involvement in $\mathrm{AD}$ although our analysis showed no difference of total monocyte number between controls and through the progression of $\mathrm{AD}$ [54-57]. With no studies focused on $\mathrm{AD}$ monocyte population shifts, our results present a progressive decline of the classical monocyte population $\left(\mathrm{CD} 14^{+} \mathrm{CD} 16^{-}\right)$with a subsequent increase in both the intermediate $\left(\mathrm{CD} 14^{+} \mathrm{CD} 16^{+}\right)$and non-classical $\left(\mathrm{CD} 14^{\text {low }} \mathrm{CD} 16^{+}\right)$subsets as disease progresses. Shifting of 
populations from the classical population, a patrolling subset, into intermediate and non-classical populations reflects monocyte maturation and pro-inflammatory activation [58]. Identification of mechanisms whereby patient monocytes are shifting to their subsets and how they are inhibited early while being activated with increasing disease burden is needed.

MDSCs are immature myeloid cells that expand under pathological conditions such as cancer and immune disorders but have yet to be fully characterized in patients or animal models of AD. These cells suppress pro-inflammatory immune cells including mature myeloid cells and $\mathrm{CD}^{+} / \mathrm{CD}^{+} \mathrm{T}$ cells. MDSCs are roughly divided into early-stage, granulocytic, and monocytic MDSCs with the latter being most suppres-

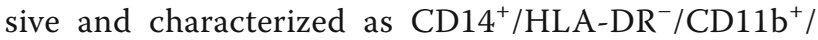
$\mathrm{CD}^{+} 3^{+}$cells $[41,59]$. MDSC numbers were increased in CDR0.5 and CDR1 patients, and decreased in CDR2/3 patients. While monocytic MDSCs are undocumented in $\mathrm{AD}$, a previous study documented an increase in granulocytic MDSCs in amnestic mild cognitive impaired patients compared with mild AD patients. However, this previous study did not provide functional data, thus it is not known whether granulocytic MDSCs have differential functions as the course of $\mathrm{AD}$ progresses or if $\mathrm{AD}$ granulocytic MDSCs have different properties compared to those of healthy controls [60].

MDSCs and mature pro-inflammatory monocytes have opposing roles in inflammation. The former provides strong immunosuppression while the latter contributes to pro-inflammatory signaling. Analyses of these phenotypes and signaling mechanisms in advancing $\mathrm{AD}$ would provide important information on the state of the immune system during the progression of AD. Additionally, these cells may directly engraft into the CNS given the evidence of a compromised $\mathrm{BBB}$ based upon the leakage of blood-derived constituents in postmortem AD brains [61-63], the increased CSF/ serum or CSF/plasma ratios of albumin [64-66], and imaging showing an age-dependent increase in BBB permeability in the hippocampus, dentate gyrus, and CA1 region [67]. Additionally, dysregulation of the neurovascular unit, responsible for maintaining BBB integrity, is known to accompany AD pathology [68]. Peripheral immune cell involvement and engraftment into the CNS are postulated to play important roles in $\operatorname{AD}[69,70]$. With that said, roles of infiltrating cells during CNS disease pathogenesis is controversial, contextual, and disease/ stage-dependent [22, 56, 71-73]. Whether and how peripheral myeloid cells dictate progression of $\mathrm{AD}$ necessitates further investigation, especially the question of peripheral immune cell migration into the CNS and other mechanisms of neuro-immune cross-talk.
Microglia and monocytes, through genetics and activation status in $\mathrm{AD}$, play a critical role in $\mathrm{AD}$ pathogenesis [6, 14-18]. Their responsibilities include phagocytosis, cytokine secretion, and restoration of homeostatic conditions with impairments to these functions being deleterious to sensitive neuronal environments in $\mathrm{AD}$. We have developed CD14 myeloid cells from control iPS cells (unpublished Zhao et al.) and polarized them to be pro-inflammatory to examine differential effects of control/AD MDSCs on activated myeloid cells. Pro-inflammatory polarization of iPS cells provides a consistent, activated cell type to compare suppression between patients without the confounding variable of primed or differentially activated mature monocytes from patients.

MDSCs from CDR0.5 patients were able to suppress pro-inflammatory IL-6 expression and protein from activated cells more than controls while CDR 1 and CDR2/3 markedly lost their suppressive function. IL- 6 transcript and protein analysis were used due to MDSCs producing no IL-6 while IL-6 is a predominant pro-inflammatory output from activated myeloid cells [74]. The IL-10 data from the RNA studies provided a mechanistic target for MDSC suppression of the myeloid cells. Adding IL-10ab to co-cultures reduced MDSC suppression of myeloid IL-6 transcripts that was most pronounced with CDR0.5 co-cultures but also observed in the other groups. IL-10ab only partially blocked IL-6 suppression leaving other additional mechanisms contributing to suppression, either through soluble signaling or direct cell contact. An extensive study into the intrinsic differences between MDSCs from prodromal and AD dementia is warranted to comprehensively parse out the predominating mechanisms of their suppressive functions and which of these are lost through AD progression.

Co-culture of MDSCs with Tresps showed increased CDR0.5 and CDR1 MDSC suppression compared with controls. The mechanism for MDSC suppression of $\mathrm{T}$ cell proliferation is through metabolism of arginine via increased Arg1 transcript in MDSCs [75, 76]. Arg1 gene expression increased in CDR0.5 MDSCs and then diminished as AD advances. Analysis of CDR1 MDSC suppression shows enhanced $\mathrm{T}$ cell proliferation but decreased M1 suppression which may suggest a suppressive function transition occurring at this stage.

Limitations of these studies involve lack of direct evidence that peripheral myeloid cells enter the CNS parenchyma and influence disease progression in $\mathrm{AD}$. Nevertheless, the definitive evidence for alterations in the $\mathrm{BBB}$ in $\mathrm{AD}$, the comparability of CSF findings and peripheral myeloid cell phenotype alterations, and the PET scan evidence of increasing neuroinflammation during the course of disease are all in accord with extensive neuro-immune cross-talk between the brain and 
peripheral immune system. Advances in neuroimaging with specialized ligands may provide tools for analyzing central vs peripheral identities as well as the anti vs pro-inflammatory phenotypes contributing to AD pathogenesis. The identification of distinct transcriptional and functional phenotypes could assist in developing these technologies [26]. Additionally, complex networks and signaling cascades involving additional cells outside our examination might assist in dictating inflammatory environments and cell phenotypes. Examination of these cells, both individually and in concert with each other, will help establish a more definitive immune environment during $\mathrm{AD}$ and through its course.

\section{Conclusions}

This study documents that peripheral monocytes are pro-inflammatory in advancing stages of $\mathrm{AD}$ but not in prodromal $\mathrm{AD}$. The pro-inflammatory responses of monocytes from prodromal $\mathrm{AD}$ patients are suppressed while advancing $\mathrm{AD}$ patients monocytes lose this suppression, and become activated and pro-inflammatory. Numbers and suppressive functions of MDSCs are increased in prodromal $\mathrm{AD}$ and decreased in patients with advancing $\mathrm{AD}$ and correlate with pro-inflammatory expression of $\mathrm{AD}$ monocytes. MDSCs also suppress Tresp which can readily enter the CNS, and loss of T effector suppression can significantly enhance inflammatory disease pathology. These findings provide a novel inflammatory paradigm that may have confounded early therapeutic interventions and provides a new basis for how future studies and treatments should be designed. Additionally, we have documented the significant impact of AD MDSCs on immune cell subsets. Understanding the role of early enhanced immunosuppression in prodromal $\mathrm{AD}$ and the subsequent dysfunction of this process in $\mathrm{AD}$ dementia may lead to novel therapeutic strategies.

\section{Additional files}

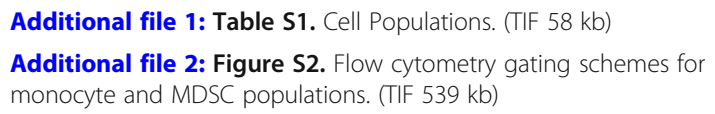

Additional file 3: Figure S9. Nanostring dataset for RNA analysis of CDR rated patients and age-matched controls. (XLSX $65 \mathrm{~kb}$ )

Additional file 4: Figure S4. IPSC-derived M1 and MDSC co-culture paradigm. (TIF $231 \mathrm{~kb}$ )

Additional file 5: Figure S3. (a) Correlation data of inflammatory RNA expression and subject age from peripheral myeloid cells. Data represent analyses of RNA expression with controls only, patients with varying levels of $A D$, and all groups combined (Control $n=20, A D n=38$ ). (b) Analyses of gender contributions to pro-inflammatory RNA expression among controls, CDR0.5, CDR1, and CDR2/3 (Control $n=10 / 10 \mathrm{M} / \mathrm{F}, \mathrm{CDR} 0.5 n=6 / 14 \mathrm{M} / \mathrm{F}$, CDR1 $n=5 / 3 \mathrm{M} / \mathrm{F}, \mathrm{CDR} 2 / 3 n=4 / 6 \mathrm{M} / \mathrm{F})$. Graphs show average \pm SEM with statistics run using two-way ANOVA with Sidak's multiple comparisons test. No statistical difference observed after age and gender data stratification unless signified by ${ }^{*} p<0.05,{ }^{* *} p<0.01$, or ${ }^{* * *} p<0.001$. (TIF $156 \mathrm{~kb}$ )
Additional file 6: Figure S6. (a) Correlation data between age and protein expression of HLADR and CD33 analyzed via flow cytometry (Control $n=30, A D n=57$ ). (b) Analyses of gender contributions to HLADR and CD33 expression on mature myeloid cells isolated from controls, CDR0.5, CDR1, and CDR2/3 (Control $n=14 / 16 \mathrm{M} / \mathrm{F}, \mathrm{CDR} 0.5 n=13 / 14 \mathrm{M} / \mathrm{F}$, CDR1 $n=8 / 10 \mathrm{M} / \mathrm{F}, \mathrm{CDR} 2 / 3 n=3 / 10 \mathrm{M} / \mathrm{F})$. Graphs show average \pm SEM with statistics run using two-way ANOVA with Sidak's multiple comparisons test. No statistical difference observed after age and gender data stratification unless signified by ${ }^{*} p<0.05$, ${ }^{* *} p<0.01$, or ${ }^{* * *} p<0.001$. (TIF $194 \mathrm{~kb}$ )

Additional file 7: Figure S5. (a) Correlation data between age and monocyte population changes. Analyses performed examined the ages of controls, varying levels of $\mathrm{AD}$, and combined groups for correlations in changes in classical monocytes, intermediate monocytes, non-classical monocytes, and MDSCs (Control $n=35, \mathrm{AD} n=66$ ). (b) Analyses of gender contributions to monocyte population changes among controls, CDR0.5, CDR1, and CDR2/3 (Control $n=20 / 15 \mathrm{M} / \mathrm{F}, \mathrm{CDR} 0.5 n=15 / 16 \mathrm{M} / \mathrm{F}, \mathrm{CDR} 1$ $n=8 / 10 \mathrm{M} / \mathrm{F}, \mathrm{CDR} 2 / 3 n=5 / 12 \mathrm{M} / \mathrm{F})$. Graphs show average \pm SEM with statistics run using two-way ANOVA with Sidak's multiple comparisons test. No statistical difference observed after age and gender data stratification unless signified by ${ }^{*} p<0.05$, ${ }^{* *} p<0.01$, or ${ }^{* * *} p<0.001$. (TIF $246 \mathrm{~kb}$ )

Additional file 8: Figure S7. (a) Analysis of gender contribution to MDSC suppressive function on pro-inflammatory M1 cells (Control $n=6 / 4 \mathrm{M}$ ) $\mathrm{F}, \mathrm{CDR} 0.5 n=5 / 6 \mathrm{M} / \mathrm{F}, \mathrm{CDR} 1 n=4 / 6 \mathrm{M} / \mathrm{F}, \mathrm{CDR} 2 / 3 n=3 / 7 \mathrm{M} / \mathrm{F})$. Graph shows average \pm SEM with statistics run using two-way ANOVA with Sidak's multiple comparisons test. No statistical difference observed after gender data stratification unless signified by ${ }^{*} p<0.05$, ${ }^{* *} p<0.01$, or ${ }^{* * *} p<0.001$. (TIF $25 \mathrm{~kb}$ )

Additional file 9: Figure S8. (a) Correlation plot graphing $T$ resp. proliferation suppression and myeloid IL-6 transcript suppression at 1:1 ratio of responding cells to MDSCs $(R=.7288 p=0.004)$. (b) IL-6 control experiment whereby MDSCs from controls $(n=6)$ and AD patients from various stages $(n=12)$ do not express IL-6 transcript when cultured alone in LPS/IFNy treatments. Corroboration with no IL-6 protein in the MDSC only treated media when analyzed via ELISA (data not shown). (TIF $29 \mathrm{~kb}$ )

\section{Acknowledgements}

We would like to thank the Stanley H. Appel Department of Neurology, the Nantz National Alzheimer Center, and Houston Methodist Hospital for their contributions and patient organization/recruitment. Individually, we acknowledge Douglas Casey, Jinghong Wang, and Neha Pal for their time and efforts in the completion of this project. We also thank patients and their families for their participation and involvement in the study.

\section{Funding}

This study was funded by the Ting Tsung and Wei Fong Chao Foundation in Houston, TX.

\section{Availability of data and materials}

Materials and/or datasets used/generated are included in the manuscript or available upon reasonable request.

\section{Author's contributions}

ADT and SHA conceived and designed research; ADT, AF, JRT, WZ, and SW performed experiments; ADT, DRB, JRT, WZ, and SHA analyzed and interpreted the data. AF, BP, and JCM recruited, organized clinical operations for sample collection, and rated/categorized patients; ADT wrote manuscript with input from all authors. All authors read and approved the final version of the manuscript.

\section{Ethics approval and consent to participate}

The study was approved by the Houston Methodist IRB and all participants signed informed consent.

\section{Consent for publication}

This manuscript has been read and approved by all authors, it has not been previously published, and is not under simultaneous consideration by another journal. Authors give consent for publication in Molecular Neurodegeneration.

\section{Competing interests}

The authors of this manuscript declare that they have no competing interests. 


\section{Publisher's Note}

Springer Nature remains neutral with regard to jurisdictional claims in published maps and institutional affiliations.

Received: 28 June 2018 Accepted: 23 October 2018 Published online: 13 November 2018

\section{References}

1. Brion JP. Neurofibrillary tangles and Alzheimer's disease. Eur Neurol. 1998;40: 130-40.

2. Hardy JA, Higgins GA. Alzheimer's disease: the amyloid cascade hypothesis. Science. 1992;256:184-5.

3. Larson EB, Kukull WA, Katzman RL. Cognitive impairment: dementia and Alzheimer's disease. Annu Rev Public Health. 1992;13:431-49.

4. Hardy J, Selkoe DJ. The amyloid hypothesis of Alzheimer's disease: progress and problems on the road to therapeutics. Science. 2002;297:353-6.

5. Holtzman DM, Morris JC, Goate AM. Alzheimer's disease: the challenge of the second century. Sci Transl Med. 2011;3:77sr71.

6. Kreisl WC, Lyoo CH, Liow JS, Wei M, Snow J, Page E, Jenko KJ, Morse $\mathrm{CL}$, Zoghbi SS, Pike WW, et al. (11)C-PBR28 binding to translocator protein increases with progression of Alzheimer's disease. Neurobiol Aging. 2016:44:53-61.

7. Akiyama H, Barger S, Barnum S, Bradt B, Bauer J, Cole GM, Cooper NR, Eikelenboom P, Emmerling M, Fiebich BL, et al. Inflammation and Alzheimer's disease. Neurobiol Aging. 2000;21:383-421.

8. Cuello AC. Early and late CNS inflammation in Alzheimer's disease: two extremes of a continuum? Trends Pharmacol Sci. 2017;38:956-66.

9. Hopperton KE, Mohammad D, Trepanier MO, Giuliano V, Bazinet RP. Markers of microglia in post-mortem brain samples from patients with Alzheimer's disease: a systematic review. Mol Psychiatry. 2018;23:177-98.

10. Wyss-Coray T, Rogers J. Inflammation in Alzheimer disease-a brief review of the basic science and clinical literature. Cold Spring Harb Perspect Med. 2012;2:a006346

11. Zimmer ER, Leuzy A, Benedet AL, Breitner J, Gauthier S, Rosa-Neto P. Tracking neuroinflammation in Alzheimer's disease: the role of positron emission tomography imaging. J Neuroinflammation. 2014;11:120

12. Schwab C, Klegeris A, McGeer PL. Inflammation in transgenic mouse models of neurodegenerative disorders. Biochim Biophys Acta. 2010;1802:889-902.

13. Wilcock DM, Zhao Q, Morgan D, Gordon MN, Everhart A, Wilson JG, Lee JE, Colton CA. Diverse inflammatory responses in transgenic mouse models of Alzheimer's disease and the effect of immunotherapy on these responses. ASN Neuro. 2011;3:249-58.

14. Bradshaw EM, Chibnik LB, Keenan BT, Ottoboni L, Raj T, Tang A, Rosenkrantz LL, Imboywa S, Lee M, Von Korff A, et al. CD33 Alzheimer's disease locus: altered monocyte function and amyloid biology. Nat Neurosci. 2013;16:848-50.

15. Griciuc A, Serrano-Pozo A, Parrado AR, Lesinski AN, Asselin CN, Mullin K, Hooli B, Choi SH, Hyman BT, Tanzi RE. Alzheimer's disease risk gene CD33 inhibits microglial uptake of amyloid beta. Neuron. 2013;78:631-43.

16. Guerreiro R, Wojtas A, Bras J, Carrasquillo M, Rogaeva E, Majounie E, Cruchaga C, Sassi C, Kauwe JS, Younkin S, et al. TREM2 variants in Alzheimer's disease. N Engl J Med. 2013;368:117-27.

17. Jonsson T, Stefansson H, Steinberg S, Jonsdottir I, Jonsson PV, Snaedal J, Bjornsson S, Huttenlocher J, Levey Al, Lah JJ, et al. Variant of TREM2 associated with the risk of Alzheimer's disease. N Engl J Med. 2013;368: 107-16.

18. Malik M, Simpson JF, Parikh I, Wilfred BR, Fardo DW, Nelson PT, Estus S. CD33 Alzheimer's risk-altering polymorphism, CD33 expression, and exon 2 splicing. J Neurosci. 2013;33:13320-5.

19. Goldeck D, Witkowski JM, Fulop T, Pawelec G. Peripheral immune signatures in Alzheimer disease. Curr Alzheimer Res. 2016:13:739-49.

20. Kipnis J. Multifaceted interactions between adaptive immunity and the central nervous system. Science. 2016;353:766-71.

21. Le Page A, Dupuis G, Frost EH, Larbi A, Pawelec G, Witkowski JM, Fulop T. Role of the peripheral innate immune system in the development of Alzheimer's disease. Exp Gerontol. 2018;107:59-66.

22. Varvel NH, Neher JJ, Bosch A, Wang W, Ransohoff RM, Miller RJ, Dingledine R. Infiltrating monocytes promote brain inflammation and exacerbate neuronal damage after status epilepticus. Proc Natl Acad Sci U S A. 2016; 113:E5665-74.
23. Carvey PM, Hendey B, Monahan AJ. The blood-brain barrier in neurodegenerative disease: a rhetorical perspective. J Neurochem. 2009;111:291-314.

24. de Vries HE, Kooij G, Frenkel D, Georgopoulos S, Monsonego A, Janigro D. Inflammatory events at blood-brain barrier in neuroinflammatory and neurodegenerative disorders: implications for clinical disease. Epilepsia. 2012:53(Suppl 6):45-52.

25. Erickson MA, Dohi K, Banks WA. Neuroinflammation: a common pathway in CNS diseases as mediated at the blood-brain barrier.

Neuroimmunomodulation. 2012;19:121-30.

26. Cronk JC, Filiano AJ, Louveau A, Marin I, Marsh R, Ji E, Goldman DH, Smirnov I, Geraci N, Acton S, et al. Peripherally derived macrophages can engraft the brain independent of irradiation and maintain an identity distinct from microglia. J Exp Med. 2018;215:1627-47.

27. Ginhoux F, Lim S, Hoeffel G, Low D, Huber T. Origin and differentiation of microglia. Front Cell Neurosci. 2013;7:45.

28. London A, Cohen M, Schwartz M. Microglia and monocyte-derived macrophages: functionally distinct populations that act in concert in CNS plasticity and repair. Front Cell Neurosci. 2013;7:34

29. Perry VH, Teeling J. Microglia and macrophages of the central nervous system: the contribution of microglia priming and systemic inflammation to chronic neurodegeneration. Semin Immunopathol. 2013;35:601-12.

30. Yang J, Zhang L, Yu C, Yang XF, Wang H. Monocyte and macrophage differentiation: circulation inflammatory monocyte as biomarker for inflammatory diseases. Biomark Res. 2014;2(1):1. https://doi.org/10.1186/ 2050-7771-2-1.

31. Ziegler-Heitbrock L, Ancuta P, Crowe S, Dalod M, Grau V, Hart DN, Leenen PJ, Liu YJ, MacPherson G, Randolph GJ, et al. Nomenclature of monocytes and dendritic cells in blood. Blood. 2010;116:e74-80.

32. Boyette LB, Macedo C, Hadi K, Elinoff BD, Walters JT, Ramaswami B, Chalasan G, Taboas JM, Lakkis FG, Metes DM. Phenotype, function, and differentiation potential of human monocyte subsets. PLoS One. 2017;12:e0176460.

33. Gawdat K, Legere S, Wong C, Myers T, Marshall JS, Hassan A, Brunt KR, Kienesberger PC, Pulinilkunnil T, Legare JF. Changes in circulating monocyte subsets (CD16 expression) and neutrophil-to-lymphocyte ratio observed in patients undergoing cardiac surgery. Front Cardiovasc Med. 2017;4:12.

34. Kim WK, Sun Y, Do H, Autissier P, Halpern EF, Piatak M Jr, Lifson JD, Burdo TH, McGrath MS, Williams K. Monocyte heterogeneity underlying phenotypic changes in monocytes according to SIV disease stage. J Leukoc Biol. 2010;87:557-67.

35. Mukherjee R, Kanti Barman P, Kumar Thatoi P, Tripathy R, Kumar Das B, Ravindran B. Non-classical monocytes display inflammatory features: validation in Sepsis and systemic lupus erythematous. Sci Rep. 2015;5:13886.

36. Ong SM, Hadadi E, Dang TM, Yeap WH, Tan CT, Ng TP, Larbi A, Wong SC. The pro-inflammatory phenotype of the human non-classical monocyte subset is attributed to senescence. Cell Death Dis. 2018:9:266.

37. Stansfield BK, Ingram DA. Clinical significance of monocyte heterogeneity. Clin Transl Med. 2015:4:5

38. Wildgruber M, Aschenbrenner T, Wendorff H, Czubba M, Glinzer A, Haller B, Schiemann M, Zimmermann A, Berger $\mathrm{H}$, Eckstein $\mathrm{HH}$, et al. The "intermediate" CD14(++)CD16(+) monocyte subset increases in severe peripheral artery disease in humans. Sci Rep. 2016;6:39483.

39. Grozdanov V, Bliederhaeuser C, Ruf WP, Roth V, Fundel-Clemens K, Zondler L, Brenner D, Martin-Villalba A, Hengerer B, Kassubek J, et al. Inflammatory dysregulation of blood monocytes in Parkinson's disease patients. Acta Neuropathol. 2014;128:651-63.

40. Zhao W, Beers DR, Hooten KG, Sieglaff DH, Zhang A, Kalyana-Sundaram S, Traini CM, Halsey WS, Hughes AM, Sathe GM, et al. Characterization of gene expression phenotype in amyotrophic lateral sclerosis monocytes. JAMA Neurol. 2017;74:677-85.

41. Bronte V, Brandau S, Chen SH, Colombo MP, Frey AB, Greten TF, Mandruzzato S, Murray PJ, Ochoa A, Ostrand-Rosenberg S, et al. Recommendations for myeloid-derived suppressor cell nomenclature and characterization standards. Nat Commun. 2016;7:12150

42. Greten TF, Manns MP, Korangy F. Myeloid derived suppressor cells in human diseases. Int Immunopharmacol. 2011;11:802-7.

43. Kong YY, Fuchsberger M, Xiang SD, Apostolopoulos V, Plebanski M. Myeloid derived suppressor cells and their role in diseases. Curr Med Chem. 2013;20: 1437-44.

44. Tamadaho RSE, Hoerauf A, Layland LE. Immunomodulatory effects of myeloid-derived suppressor cells in diseases: role in cancer and infections. Immunobiology. 2018;223:432-42. 
45. Salminen A, Kaarniranta K, Kauppinen A. The potential importance of myeloid-derived suppressor cells (MDSCs) in the pathogenesis of Alzheimer's disease. Cell Mol Life Sci. 2018;75:3099. https://doi.org/10.1007/ s00018-018-2844-6.

46. Albert MS, DeKosky ST, Dickson D, Dubois B, Feldman HH, Fox NC, Gamst A, Holtzman DM, Jagust WJ, Petersen RC, et al. The diagnosis of mild cognitive impairment due to Alzheimer's disease: recommendations from the National Institute on Aging-Alzheimer's Association workgroups on diagnostic guidelines for Alzheimer's disease. Alzheimers Dement. 2011;7:270-9.

47. McKhann GM, Knopman DS, Chertkow H, Hyman BT, Jack CR Jr, Kawas CH, Klunk WE, Koroshetz WJ, Manly JJ, Mayeux R, et al. The diagnosis of dementia due to Alzheimer's disease: recommendations from the National Institute on Aging-Alzheimer's Association workgroups on diagnostic guidelines for Alzheimer's disease. Alzheimers Dement. 2011;7:263-9.

48. Yanagimachi MD, Niwa A, Tanaka T, Honda-Ozaki F, Nishimoto S, Murata Y, Yasumi T, Ito J, Tomida S, Oshima K, et al. Robust and highly-efficient differentiation of functional monocytic cells from human pluripotent stem cells under serum- and feeder cell-free conditions. PLoS One. 2013:8:e59243.

49. Guillemin GJ, Brew BJ. Microglia, macrophages, perivascular macrophages, and pericytes: a review of function and identification. J Leukoc Biol. 2004;75: 388-97

50. Prinz M, Priller J. Microglia and brain macrophages in the molecular age: from origin to neuropsychiatric disease. Nat Rev Neurosci. 2014;15:300-12.

51. Yamasaki R, Lu H, Butovsky O, Ohno N, Rietsch AM, Cialic R, Wu PM, Doykan CE, Lin J, Cotleur AC, et al. Differential roles of microglia and monocytes in the inflamed central nervous system. J Exp Med. 2014;211:1533-49.

52. Meyer PF, Savard M, Poirier J, Labonte A, Rosa-Neto P, Weitz TM, Town T, Breitner J. Alzheimer's disease neuroimaging I, group P-AR: bi-directional Association of Cerebrospinal Fluid Immune Markers with stage of Alzheimer's disease pathogenesis. J Alzheimers Dis. 2018;63:577-90.

53. Fan Z, Brooks DJ, Okello A, Edison P. An early and late peak in microglial activation in Alzheimer's disease trajectory. Brain. 2017;140:792-803.

54. Feng Y, Li L, Sun XH. Monocytes and Alzheimer's disease. Neurosci Bull. 2011;27:115-22.

55. Martin E, Boucher C, Fontaine B, Delarasse C. Distinct inflammatory phenotypes of microglia and monocyte-derived macrophages in Alzheimer's disease models: effects of aging and amyloid pathology. Aging Cell. 2017;16:27-38.

56. Saresella M, Marventano I, Calabrese E, Piancone F, Rainone V, Gatti A, Alberoni M, Nemni R, Clerici M. A complex proinflammatory role for peripheral monocytes in Alzheimer's disease. J Alzheimers Dis. 2014;38:403-13.

57. Theriault $P$, ElAli A, Rivest $S$. The dynamics of monocytes and microglia in Alzheimer's disease. Alzheimers Res Ther. 2015;7:41

58. Wong KL, Tai JJ, Wong WC, Han H, Sem X, Yeap WH, Kourilsky P, Wong SC. Gene expression profiling reveals the defining features of the classical, intermediate, and nonclassical human monocyte subsets. Blood. 2011;118: e16-31

59. Youn Jl, Gabrilovich DI. The biology of myeloid-derived suppressor cells: the blessing and the curse of morphological and functional heterogeneity. Eur J Immunol. 2010;40:2969-75.

60. Le Page A, Garneau H, Dupuis G, Frost EH, Larbi A, Witkowski JM, Pawelec G, Fulop T. Differential phenotypes of myeloid-derived suppressor and T regulatory cells and cytokine levels in amnestic mild cognitive impairment subjects compared to mild Alzheimer diseased patients. Front Immunol. 2017;8:783.

61. Wisniewski HM, Kozlowski PB. Evidence for blood-brain barrier changes in senile dementia of the Alzheimer type (SDAT). Ann N Y Acad Sci. 1982;396: 119-29.

62. Wisniewski HM, Vorbrodt AW, Wegiel J. Amyloid angiopathy and bloodbrain barrier changes in Alzheimer's disease. Ann N Y Acad Sci. 1997;826: $161-72$.

63. Zipser BD, Johanson CE, Gonzalez L, Berzin TM, Tavares R, Hulette CM, Vitek MP, Hovanesian V, Stopa EG. Microvascular injury and blood-brain barrier leakage in Alzheimer's disease. Neurobiol Aging. 2007;28:977-86.

64. Alafuzoff I, Adolfsson R, Bucht G, Winblad B. Albumin and immunoglobulin in plasma and cerebrospinal fluid, and blood-cerebrospinal fluid barrier function in patients with dementia of Alzheimer type and multi-infarct dementia. J Neurol Sci. 1983;60:465-72.
65. Blennow K, Wallin A, Fredman P, Karlsson I, Gottfries CG, Svennerholm L. Blood-brain barrier disturbance in patients with Alzheimer's disease is related to vascular factors. Acta Neurol Scand. 1990;81:323-6.

66. Skoog I, Wallin A, Fredman P, Hesse C, Aevarsson O, Karlsson I, Gottfries CG, Blennow K. A population study on blood-brain barrier function in 85-yearolds: relation to Alzheimer's disease and vascular dementia. Neurology. 1998;50:966-71.

67. Montagne A, Barnes SR, Sweeney MD, Halliday MR, Sagare AP, Zhao Z, Toga AW, Jacobs RE, Liu CY, Amezcua L, et al. Blood-brain barrier breakdown in the aging human hippocampus. Neuron. 2015;85:296-302.

68. Yamazaki Y, Kanekiyo T. Blood-brain barrier dysfunction and the pathogenesis of Alzheimer's disease. Int J Mol Sci. 2017;18.

69. Prinz M, Priller J. The role of peripheral immune cells in the CNS in steady state and disease. Nat Neurosci. 2017;20:136-44.

70. Rezai-Zadeh K, Gate D, Town T. CNS infiltration of peripheral immune cells: D-day for neurodegenerative disease? J Neurolmmune Pharmacol. 2009;4: 462-75.

71. El Khoury J, Toft M, Hickman SE, Means TK, Terada K, Geula C, Luster AD. $\mathrm{Ccr} 2$ deficiency impairs microglial accumulation and accelerates progression of Alzheimer-like disease. Nat Med. 2007;13:432-8.

72. Harms AS, Delic V, Thome AD, Bryant N, Liu Z, Chandra S, Jurkuvenaite A, West AB. Alpha-Synuclein fibrils recruit peripheral immune cells in the rat brain prior to neurodegeneration. Acta Neuropathol Commun. 2017;5:85.

73. Harms AS, Thome AD, Yan Z, Schonhoff AM, Williams GP, Li X, Liu Y, Qin H, Benveniste EN, Standaert DG. Peripheral monocyte entry is required for alpha-Synuclein induced inflammation and neurodegeneration in a model of Parkinson disease. Exp Neurol. 2018;300:179-87.

74. Beury DW, Parker KH, Nyandjo M, Sinha P, Carter KA, Ostrand-Rosenberg S. Cross-talk among myeloid-derived suppressor cells, macrophages, and tumor cells impacts the inflammatory milieu of solid tumors. J Leukoc Biol. 2014;96:1109-18.

75. Bronte $\mathrm{V}$, Zanovello P. Regulation of immune responses by L-arginine metabolism. Nat Rev Immunol. 2005:5:641-54

76. Rodriguez PC, Ochoa AC. Arginine regulation by myeloid derived suppressor cells and tolerance in cancer: mechanisms and therapeutic perspectives. Immunol Rev. 2008;222:180-91.

\section{Ready to submit your research? Choose BMC and benefit from:}

- fast, convenient online submission

- thorough peer review by experienced researchers in your field

- rapid publication on acceptance

- support for research data, including large and complex data types

- gold Open Access which fosters wider collaboration and increased citations

- maximum visibility for your research: over $100 \mathrm{M}$ website views per year

At BMC, research is always in progress.

Learn more biomedcentral.com/submissions 